Portland State University

PDXScholar

Electrical and Computer Engineering Faculty

Publications and Presentations

6-1-1985

\title{
Spontaneous coherent pulsations in ring-laser oscillators: stability criteria
}

Lee W. Casperson

Portland State University

Follow this and additional works at: https://pdxscholar.library.pdx.edu/ece_fac

Part of the Electrical and Computer Engineering Commons

Let us know how access to this document benefits you.

\section{Citation Details}

Lee W. Casperson, "Spontaneous coherent pulsations in ring-laser oscillators: stability criteria," J. Opt. Soc. Am. B 2, 993-997 (1985).

This Article is brought to you for free and open access. It has been accepted for inclusion in Electrical and Computer Engineering Faculty Publications and Presentations by an authorized administrator of PDXScholar. Please contact us if we can make this document more accessible: pdxscholar@pdx.edu. 


\title{
Spontaneous coherent pulsations in ring-laser oscillators: stability criteria
}

\author{
Lee W. Casperson \\ Department of Electrical Engineering, Portland State University, Portland, Oregon 97207
}

Received November 5, 1984; accepted January 2, 1985

\begin{abstract}
The stability criteria implied by a simplified semiclassical laser model are considered in detail. It is found that two types of criteria must be distinguished. The first concerns the smallest pump rate for which infinitesimal perturbations of the steady-state solution will increase with time, and the second concerns the smallest pump rate for which large amplitude modulations will not damp away.
\end{abstract}

\section{INTRODUCTION}

It is now well known that the semiclassical laser equations can under some conditions predict unstable laser behavior with resulting output pulsations that are periodic or chaotic. $\mathrm{Al}$ though some aspects of these instabilities can be understood analytically, the complexity of the equations often requires numerical solutions for a reasonably rigorous description. Numerical solutions of the equations for a single-mode homogeneously broadened laser have been known for many years. ${ }^{1-4}$ Recently, numerical solutions for inhomogeneously broadened lasers have also been obtained. ${ }^{5-11}$ These numerical models seem now to be sufficiently accurate to provide quantitative agreement with experimental data. ${ }^{8}$

Unfortunately, the numerical solutions for any particular laser are somewhat difficult and costly to carry out. Also, they generally contain so many physical parameters that, once obtained, they cannot be applied to any other type of laser. Thus there would be value in finding some type of graphical representation that, even though approximate, might provide insight into the behavior of broad classes of lasers. The most basic results of this type are the stability criteria, and such criteria have long been known for homogeneously ${ }^{12-29}$ and, more recently, inhomogeneously ${ }^{30-38}$ broadened lasers. A brief historical review of the development of these criteria is included in Ref. 6.

In a graphical representation, the stability criteria are curves that show the ranges of parameters for which the laser will be stable and produce a cw output and the ranges for which the output will consist of pulsations. The criteria usually do not, however, provide direct information on the pulsation wave forms, damping rates, chaoticness, etc. Unfortunately, only a rather small number of parameter variations can be represented by a reasonable number of twodimensional graphs. Therefore the laser equations must be simplified sufficiently at the outset so that a graphical representation of the stability criteria is useful, and only a few isolated cases have been covered by previous graphical results. The purpose of this study is to develop a somewhat more comprehensive set of stability curves for a laser model that is describable with four parameters including the phase decay rate, population-difference decay rate, electric-field decay rate, and threshold parameter. These curves are obtained for the two limits of homogeneous and inhomogeneous line broadening.
An important aspect of the laser equations is that for any given set of conditions the equations may possess several (or infinitely many) different solutions. One of these solutions is static (besides the trivial zero-field solution), and the rest are dynamic. Some of the solutions are stable in the sense that perturbations away from the solutions decay away, and some are unstable in the sense that perturbations grow. In this study we have emphasized two of the basic stability criteria associated with the nontrivial steady-state solution. One of these criteria, which we will call type 1 , corresponds to the minimum value of the threshold parameter for which a small perturbation away from steady-state grows into an oscillatory solution. The other criterion corresponds to the minimum value of the threshold parameter for which large-amplitude oscillatory solutions do not decay to the steady-state solution. That these criteria might not be the same has been noted by Minden,33 Minden and Casperson, ${ }^{38}$ and Zeghlache and Mandel.4 As a consequence of the difference between the stability criteria, the stability of the steady-state solution depends on the magnitude of the perturbations to which it is subjected. A large perturbation may cause the onset of pulsations, whereas a small perturbation may not.

The basic equations on which this study is based are briefly reviewed in Section 2. The stability criteria for the homogeneous limit are discussed in Section 3, and it is found that the type 2 instability threshold may be far below the more familiar type 1 threshold. The stability criteria for the inhomogeneous limit are considered in Section 4, and in this case the two types of instability threshold seem to be equal to each other.

\section{THEORY}

The semiclassical dynamical equations for a single-mode oscillator have been given in many places. The starting point here is the normalized unidirectional gas-laser set given as Eqs. (21)-(25) in Ref. 9. These equations are

$$
\begin{aligned}
& \frac{\partial P_{r}}{\partial t}=-\gamma\left[P_{r}+(y-V) P_{i}-A_{i} D\right], \\
& \frac{\partial P_{i}}{\partial t}=-\gamma\left[P_{i}-(y-V) P_{r}+A_{r} D\right], \\
& \frac{\partial D}{\partial t}=\lambda_{d}-\gamma_{d}\left(D-A_{r} P_{i}+A_{i} P_{r}\right),
\end{aligned}
$$




$$
\begin{aligned}
& \frac{\partial A_{r}}{\partial t}=-\gamma_{c}\left[A_{r}+\delta\left(y-y_{0}\right) A_{i}+\int_{-\infty}^{\infty} P_{i} \mathrm{~d} V\right], \\
& \frac{\partial A_{i}}{\partial t}=-\gamma_{c}\left[A_{i}-\delta\left(y-y_{0}\right) A_{r}-\int_{-\infty}^{\infty} P_{r} \mathrm{~d} V\right] .
\end{aligned}
$$

The dependent variables in Eqs. (1)-(5) include the real part of the velocity-dependent polarization $P_{r}(V)$ and the imaginary part $P_{i}(V)$, the difference between the velocity-dependent populations of the upper and lower laser levels $D(V)$, the real part of the electric field $A_{r}$, and the imaginary part $A_{i}$. The velocity-dependent pumping rate is $\lambda_{d}(V)$, and the decay rates include the polarization decay rate $\gamma$, the population decay rate $\gamma_{d}$, and the electric-field cavity decay rate $\gamma_{c}$. The parameter $y$ represents the normalized frequency difference between the actual lasing frequency and line center, and $y_{o}$ represents the corresponding parameter value when dispersion is neglected. The parameter $\delta=\gamma / \gamma_{c}$ represents the ratio of the polarization decay rate to the electric-field decay rate. Whereas a more rigorous model would include other physical effects, it has been shown that Eqs. (1)-(5) provide at least a qualitative description of most of the laser phenomena of interest.

Unfortunately, Eqs. (1)-(5) still contain too many parameters for obtaining generally useful stability criteria, and a further restriction is necessary. If it is assumed that the laser is tuned to line center $\left(y=y_{0}=0\right)$, Eqs. (1)-(5) reduce to

$$
\begin{aligned}
& \frac{\partial P_{r}}{\partial t}=-\gamma\left(P_{r}-V P_{i}-A_{i} D\right), \\
& \frac{\partial P_{i}}{\partial t}=-\gamma\left(P_{i}+V P_{r}+A_{r} D\right) \\
& \frac{\partial D}{\partial t}=\lambda_{d}-\gamma_{d}\left(D-A_{r} P_{i}+A_{i} P_{r}\right), \\
& \frac{\partial A_{r}}{\partial t}=-\gamma_{c}\left(A_{r}+\int_{-\infty}^{\infty} P_{i} \mathrm{~d} V\right) \\
& \frac{\partial A_{i}}{\partial t}=-\gamma_{c}\left(A_{i}-\int_{-\infty}^{\infty} P_{r} \mathrm{~d} V\right) .
\end{aligned}
$$

In most gas lasers the pumping rate is assumed to be a Gaussian function of velocity. Then the real part of the polarization can be assumed to be an odd function of velocity, and the imaginary part of the electric field can be set equal to zero. In homogeneously broadened lasers tuned to line center this type of reduction is always possible and may be related to the phase difference between the sideband perturbations and the central mode. ${ }^{39}$ Equations (6)-(10) now reduce to

$$
\begin{aligned}
& \frac{\partial P_{r}}{\partial t}=-\gamma\left(P_{r}-V P_{i}\right), \\
& \frac{\partial P_{i}}{\partial t}=-\gamma\left(P_{i}+V P_{r}+A_{r} D\right), \\
& \frac{\partial D}{\partial t}=\lambda_{d}-\gamma_{d}\left(D-A_{r} P_{i}\right), \\
& \frac{\partial A_{r}}{\partial t}=-\gamma_{c}\left(A_{r}+\int_{-\infty}^{\infty} P_{i} \mathrm{~d} V\right) .
\end{aligned}
$$

This is the simplest useful form of the semiclassical laser equations for a single-mode laser with line-center tuning and arbitrary mixed line broadening. The following sections consider the stability criteria that are implied by these equations in the two limits of homogeneous and inhomogeneous line broadening.

\section{HOMOGENEOUS BROADENING}

In a homogeneously broadened laser, Eqs. (11)-(14) simplify substantially. This limit is obtained by integrating Eqs. (11)-(13) over velocity while remembering that $V$ is always small. The result of this procedure is that Eq. (11) becomes irrelevant, and the remaining equations reduce to

$$
\begin{aligned}
& \frac{\partial P_{t}}{\partial t}=-\gamma\left(P_{t}+A_{r} D_{t}\right), \\
& \frac{\partial D_{t}}{\partial t}=-\gamma_{d}\left(D_{t}-r-A_{r} P_{t}\right), \\
& \frac{\partial A_{r}}{\partial t}=-\gamma_{c}\left(A_{r}+P_{t}\right),
\end{aligned}
$$

where the total polarization is given by $P_{t}=\int_{-\infty}^{\infty} P_{i} \mathrm{~d} V$, the total pump rate is $\lambda_{t}=\int_{-\infty}^{\infty} \lambda_{d} \mathrm{~d} V$, and the total population difference is $D_{t}=\int_{-\infty}^{\infty} D \mathrm{~d} V$. We have also introduced in Eq. (16) the parameter $r=\lambda_{t} / \gamma_{d}$.

Equations (15)-(17) are simple enough that one of the threshold criteria can be obtained analytically, and the corresponding analyses of similar equations have been discussed in most of Refs. 12-29. The steady-state solution is obtained by setting the time derivatives to zero, and in this case the equations are

$$
\begin{aligned}
& P_{t s}=-A_{r s} D_{t s}, \\
& D_{t s}=r+A_{r s} P_{t s}, \\
& A_{r s}=-P_{t s} .
\end{aligned}
$$

When these equations are combined, one finds that the steady-state normalized population difference is $D_{t s}=1$, and the steady-state intensity is $A_{r s}{ }^{2}=r-1$. Thus the threshold parameter $r$ measures the amount by which the normalized pump rate exceeds unity.

The perturbation stability (type 1 stability) of the steadystate solution can be studied by linearizing the equations with the substitutions

$$
\begin{aligned}
& P_{t}=P_{t s}+P^{\prime}, \\
& D_{t}=D_{t s}+D^{\prime}, \\
& A_{r}=A_{r s}+A^{\prime},
\end{aligned}
$$

where the primed quantities are assumed to be much smaller than the corresponding steady-state values. There are now two ways in which to proceed. One may assume complex exponential time dependences for the dependent variables in the linearized equations [i.e., $\exp (s t)$, with $s$ complex]. This substitution leads to an equation in the complex frequency $s$, and the stability boundary is obtained from the condition that $s$ is purely imaginary. Alternatively, if one is interested only in the instability threshold, it may be more straightforward to assume at the outset that the perturbations have a purely oscillatory form with no net gain [i.e., $\exp (i \omega t)$, with $\omega$ reall ${ }^{30,31}$ These two approaches yield the same threshold condition, and for Eqs. (15)-(17) that condition is

$$
r_{t h}=1+\frac{\left(\gamma+\gamma_{d}+\gamma_{c}\right)\left(\gamma+\gamma_{c}\right)}{\gamma\left(\gamma_{c}-\gamma-\gamma_{d}\right)}
$$




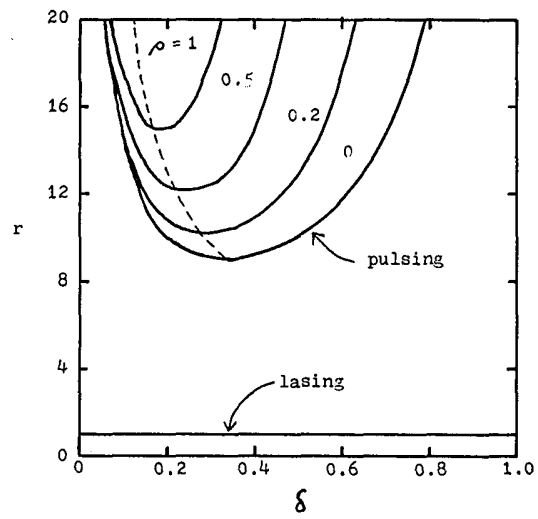

Fig. 1. Perturbation stability criteria (type 1) for homogeneously broadened lasers as a function of the ratio $\delta=\gamma / \gamma_{c}$ for various values of the ratio $\rho=\gamma_{d} / \gamma$. The dashed line is a plot of Eq. (29). The lowest possible value of the threshold parameter for instability in this model is $r=9$ at the parameter values $\rho=0$ and $\delta=1 / 3$.

provided that the condition

$$
\gamma_{c}>\gamma+\gamma_{d}
$$

(or $r_{t h}>1$ ) is also satisfied. The radian frequency of the infinitesimal oscillations at the instability threshold can also be found, and the result is

$$
\omega=\left[\frac{2 \gamma_{d} \gamma_{c}\left(\gamma+\gamma_{c}\right)}{\gamma_{c}-\gamma-\gamma_{d}}\right]^{1 / 2}
$$

In its present form the stability threshold given in Eq. (24) contains too many variables for easy graphical display. We find it convenient here and in Section 4 to introduce the dimensionless decay rate ratios $\delta=\gamma / \gamma_{c}$ and $\rho=\gamma_{d} / \gamma$. With these definitions, Eq. (24) and condition (25) reduce to

$$
r_{t h}=1+\frac{(1+\delta+\rho \delta)(1+\delta)}{\delta(1-\delta-\rho \delta)}
$$

and

$$
\delta<(1+\rho)^{-1} .
$$

Equation (27) can be readily plotted, and typical curves are given in Fig. 1. For some applications it would be of interest to know the value of $\delta$ at which the threshold parameter has its minimum value. That question can be answered by differentiating Eq. (27) with respect to $\delta$, and the result is

$$
\delta=\frac{-1-\rho+\left(4+5 \rho+2 \rho^{2}\right)^{1 / 2}}{3+4 \rho+\rho^{2}} .
$$

The corresponding value of the threshold parameter is $6,20-23$

$$
r_{t h}=5+3 \rho+2\left(4+6 \rho+2 \rho^{2}\right)^{1 / 2} .
$$

Equation (29) is plotted as a dashed line in Fig. 1.

It would seem from Fig. 1 that spontaneous pulsations would be possible only in a laser that is operating far above threshold. The lowest threshold parameter for spontaneous pulsations that is allowed by Eq. (30) is $r_{t h}=9$, and this value is obtained with $\rho=0$ and $\delta=1 / 3$. In fact, however, this result can be misleading. The second type of threshold parameter that we are considering has entirely different threshold curves. As mentioned above, this other threshold condition corresponds to a laser that is initially pulsing. The threshold parameter is then gradually reduced until the pul- sations cease. This second type of threshold is most easily obtained directly from time-domain numerical computations based on Eqs. (15)-(17). The new criteria corresponding to the same parameters used in Fig. 1 are shown in Fig. 2. It is apparent from a comparison of Figs. 1 and 2 that the second type of threshold can be much lower than the first type. Thus it would not be so difficult as one might suppose to obtain a continuous train of pulsations from a homogeneously broadened laser, provided that one could introduce an appropriately strong starting transient. Such transients occur naturally in lasers with gain or loss switching and in those with relatively high levels of quantum noise or technical noise owing to mirror vibrations, etc.

Another significant feature of curves such as those shown in Fig. 2 that is for small values of $\rho$ spontaneous pulsations can be obtained with values of $\delta$ greater than unity. This is contrary to the often quoted results obtained from perturbation analyses and indicated here in inequality (28). For small values of $\rho$ the output pulsations consist of infinite trains of narrow spikes, and typical intensity and inversion waveforms (with $\rho=0.01, \delta=1.1$, and $r=15$ ) are shown in Fig. 3 .

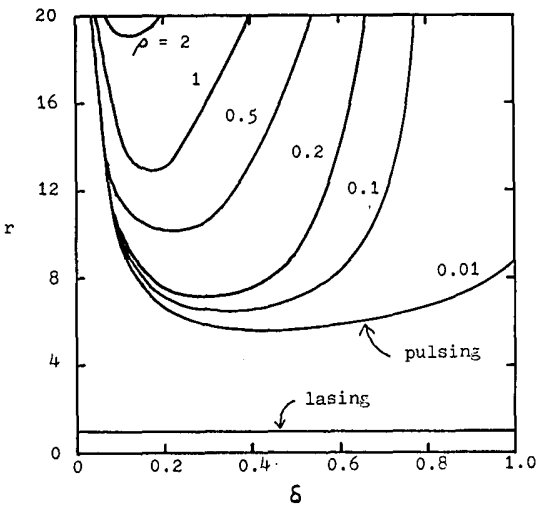

Fig. 2. Large amplitude stability criteria (type 2) for homogeneously broadened lasers as a function of the ratio $\delta=\gamma / \gamma_{\mathrm{c}}$ for various values of the ratio $\rho=\gamma_{d} / \gamma$. It is clear from this result that the type 2 stability threshold can be much less than the value $r=9$ found from perturbational analyses, and undamped pulsations are also possible for $\delta>1$.

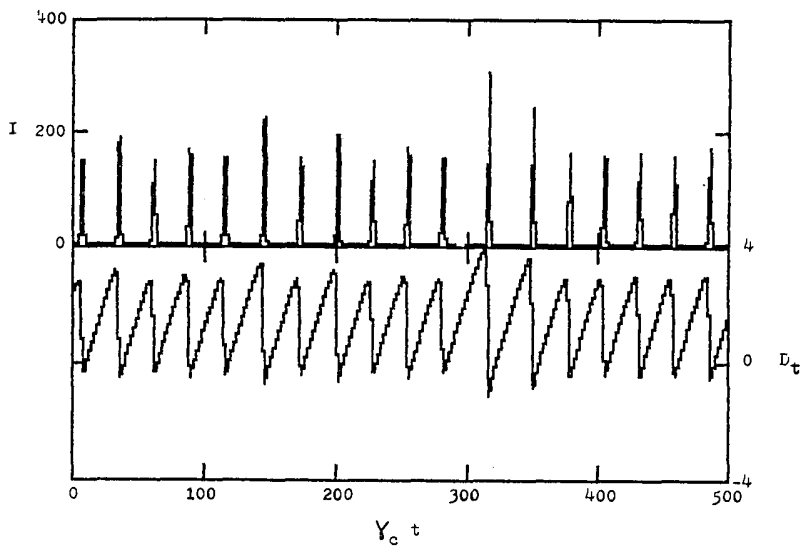

Fig. 3. Undamped output spiking in a homogeneously broadened laser with $\rho=0.01, \delta=1.1$, and $r=15$. The intensity pulse heights ( $I=A_{r}{ }^{2}$ ) are represented accurately in the top half of this figure, but the widths are shown more clearly in Fig. 4. The time-dependent population difference $D_{t}$ is shown in the bottom half, and it is significant that with this semiclassical model the population difference routinely takes on negative values during and after the intensity spikes. 


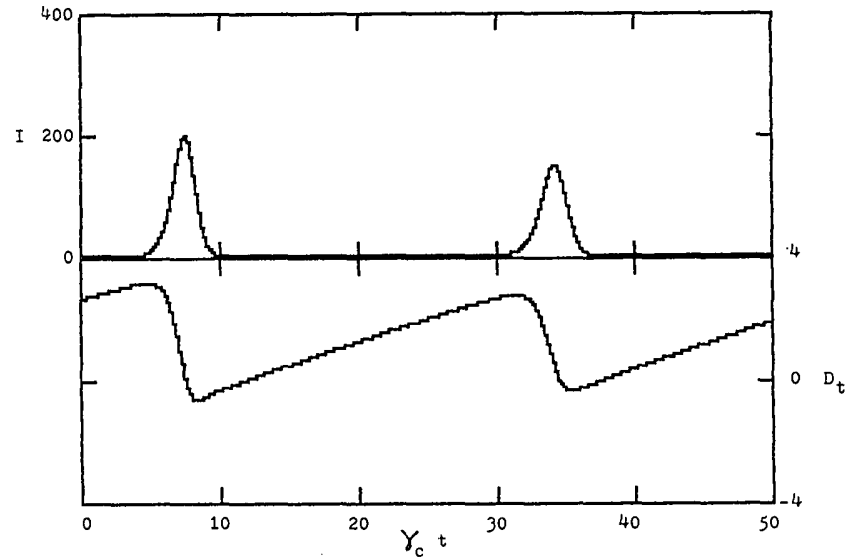

Fig. 4. Expanded version of two pulses from an undamped spiking waveform like that shown in Fig. 3 with $\rho=0.01, \delta=1.1$, and $r=15$. Again, the intensity is plotted in the top half and the population difference is shown in the bottom half.

The actual form of the intensity spikes is seen more clearly with an expanded time scale as illustrated in Fig. 4. During each spike the population difference may be driven to a large negative value, and the resulting absorption causes the interpulse field amplitude to become very small. This behavior seems not to have been noted previously and contrasts directly with rate-equation analyses. In rate-equation models, saturation cannot cause a negative population difference. With a somewhat more general semiclassical model, the spiking phenomena described here may be related to the well-known spiking effects that occur in solid-state lasers.

Although the emphasis here is, of course, on the laser equations, the relationship of these results to another formalism may also be noted. Thus, if one introduces the new parameters $t^{\prime}=\gamma t, \sigma=\gamma_{c} / \gamma$, and $b=\gamma_{d} / \gamma$ together with the new dependent variables $x=b^{1 / 2} A_{r}, y=-b^{1 / 2} P_{t}$, and $z=r$ $-D_{t}$, Eqs. (15)-17 take the forms

$$
\begin{aligned}
& \frac{\partial x}{\partial t^{\prime}}=-\sigma(x-y), \\
& \frac{\partial y}{\partial t^{\prime}}=-y+r x-x z, \\
& \frac{\partial z}{\partial t^{\prime}}=-b z+x y .
\end{aligned}
$$

These equations were introduced by Lorenz in his study of convection in fluids, ${ }^{40}$ and they have found other applications as well. For the Lorenz model, the stability criteria given in Eq. (24) and condition (25) become

$$
\begin{aligned}
& r_{t h}=\frac{\sigma(\sigma+b+3)}{\sigma-b-1}, \\
& \sigma>b+1 .
\end{aligned}
$$

That pulsating solutions are still possible for $r$ values less than $r_{t h}$ was discussed previously, ${ }^{41}$ but, as noted above, inequality (35) is also unnecessary for the occurrence of pulsations.

\section{INHOMOGENEOUS BROADENING}

The laser equations do not simplify so much in the inhomogeneous limit as they do in the homogeneous limit. Nevertheless, it is helpful to transform them a bit so that the threshold parameter is included explicitly. First we introduce the Gaussian velocity dependence of the pump rate and the population difference using the substitutions

$$
\begin{aligned}
\lambda_{d} & =\lambda_{0} \exp \left(-\epsilon^{2} V^{2}\right), \\
D & =D_{0} \exp \left(-\epsilon^{2} V^{2}\right)+D^{\prime}(V),
\end{aligned}
$$

where the parameter $\epsilon$ measures the relative widths of the homogeneous and inhomogeneous line shapes and $D_{0}$ indicates the unsaturated population difference. If Eqs. (36) and (37) are inserted into Eqs. (11)-(14) one obtains

$$
\begin{aligned}
& \frac{\partial P_{r}}{\partial t}=-\gamma\left(P_{r}-V P_{i}\right), \\
& \frac{\partial P_{i}}{\partial t}=-\gamma\left(P_{i}+V P_{r}+A_{r} D_{0}+A_{r} D^{\prime}\right), \\
& \frac{\partial D^{\prime}}{\partial t}=-\gamma_{d}\left(D^{\prime}-A_{r} P_{i}\right), \\
& \frac{\partial A_{r}}{\partial t}=-\gamma_{c}\left(A_{r}+\int_{-\infty}^{\infty} P_{i} d V\right),
\end{aligned}
$$

where $D_{0}$ has been set equal to $\lambda_{0} / \gamma_{d}$ and $\epsilon$ has been set to zero (the inhomogeneous limit).

The steady-state version of Eqs. (38)-(41) is

$$
\begin{aligned}
& P_{r s}=V P_{i s}, \\
& P_{i s}=-V P_{r s}-A_{r s} D_{0}-A_{r s} D_{s}^{\prime}, \\
& D_{s}^{\prime}=A_{r s} P_{i s}, \\
& A_{r s}=-\int_{-\infty}^{\infty} P_{i s} \mathrm{~d} V .
\end{aligned}
$$

Equations (42)-(44) may be combined to obtain

$$
P_{i s}=\frac{-A_{r s} D_{0}}{1+V^{2}+A_{r s}^{2}},
$$

and with Eq. (45) this is

$$
\begin{aligned}
1 & =D_{0} \int_{-\infty}^{\infty} \frac{\mathrm{d} V}{1+V^{2}+A_{r s}^{2}} \\
& =D_{0} \pi\left(1+A_{r s}^{2}\right)^{-1 / 2} .
\end{aligned}
$$

Since the unsaturated population difference is $D_{0}=\pi^{-1}(1+$ $\left.A_{r s}^{2}\right)^{1 / 2}$ and the threshold population difference is $\pi^{-1}$, it follows that the threshold parameter for an inhomogeneously broadened laser should be defined as $r=\pi D_{0}$. With this

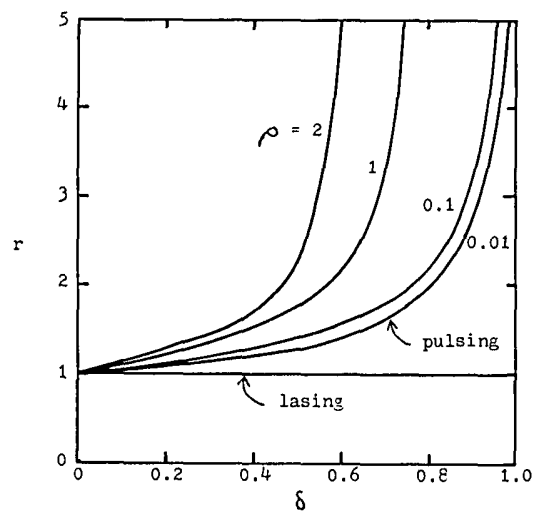

Fig. 5. Stability criteria for inhomogeneously broadened lasers as a function of the ratio $\delta=\gamma / \gamma_{c}$ for various values of the ratio $\rho=$ $\gamma_{d} / \gamma$ 
understanding, Eqs. (38)-(41) governing an inhomogeneously broadened laser are completely analogous to Eqs. (15)-(17) for a homogeneously broadened laser.

The stability of Eqs. (38)-(41) against small amplitude perturbations can be studied by using almost the same methods that have been used for the homogeneously broadened laser. Thus the equations may be linearized about the steady-state solution, and the results may then be integrated over velocity and transformed into a polynomial equation for the complex frequency. This approach was introduced for the stability analysis of rate-equation models of inhomogeneously broadened lasers, ${ }^{42}$ but it is also effective for semiclassical models. ${ }^{35-37}$ Alternatively, one can derive the threshold conditions from the gain and phase constraints on the first-order modulation sidebands. ${ }^{30-34,38}$ Whereas these procedures are straightforward in principle, they are sufficiently complicated that for present purposes we have chosen instead to derive the stability curves from direct numerical solutions of the differential equations.

A set of stability curves for small amplitude perturbations of an inhomogeneously broadened laser is shown in Fig. 5 . The most obvious feature of these curves is that the pulsation threshold can be much lower for an inhomogeneously broadened laser than for a homogeneously broadened laser, as represented in Fig. 1. In fact it is clear from Fig. 5 that for small values of $\delta$ spontaneous pulsations occur whenever the laser is significantly above the ordinary oscillation threshold $r=1$.

Based on the results obtained above for homogeneously broadened lasers, one might expect that lower pulsation thresholds would result if the laser were started in a pulsating condition and then the threshold parameter were reduced until the pulsations ceased. However, for the range and resolution of our computations to date, these two threshold types seem to be identical. It may be noted too that, in contrast to the homogeneous broadening limit, the threshold contours seem always to remain below $\delta=1$.

\section{SUMMARY}

The semiclassical laser equations predict the occurrence of spontaneous pulsations for wide ranges of parameter values. Since the actual computation of pulsation waveforms is somewhat complicated, it has seemed worthwhile to develop graphical representations of the stability criteria for wide ranges of field, polarization, and population lifetimes in the two limits of homogeneous and inhomogeneous line broadening. We find that in the homogeneous limit (and in cases of mixed line broadening) it is necessary to distinguish two types of instability threshold. The first type, which is most familiar, is defined by the condition that an infinitesimal sideband on the cw oscillation mode have gain exactly equal to loss. The second type of instability threshold corresponds to the minimum value of the threshold parameter for which a strongly pulsating field will not damp away to the corresponding steady-state solution. It is found that this second type of threshold may be much lower than the first, and hence the onset of pulsations in a cw laser oscillator with substantial homogeneous line broadening is strongly dependent on the magnitude of any perturbations. Another significant conclusion is that spontaneous pulsations can sometimes occur with a cavity decay rate $\gamma_{c}$ that is smaller than the polarization decay rate $\gamma$, contrary to most previous discussions.

\section{REFERENCES}

1. E. R. Buley and F. W. Cummings, Phys. Rev. 134, A1454 (1964).

2. M. Mayr, H. Risken, and H. D. Vollmer, Opt. Commun. 36, 480 (1981).

3. L. W. Casperson, presented at the third New Zealand Symposium on Laser Physics, January 17-23, 1983, Hamilton, New Zealand.

4. H. Zeghlache and P. Mandel, J. Opt. Soc. Am. B 2, 18 (1985).

5. L. W. Casperson, IEEE J. Quantum Electron. QE-14, 756 (1978).

6. L. W. Casperson, in Lecture Notes in Physics, D. Walls and J. Harvey, eds. (Springer-Verlag, Berlin, 1983), pp. 88-106.

7. R. Graham and Y. Cho, Opt. Commun. 47, 52 (1983).

8. L. W. Casperson, J. Opt. Soc. Am. B 2, 62 (1985).

9. L. W. Casperson, J. Opt. Soc. Am. B 2, 73 (1985).

10. P. W. Milonni, Department of Physics, University of Arkansas, Fayetteville, Arkansas 72701, J. R. Ackerhalt, and M.-L. Shih (personal communication).

11. M.-L. Shih, P. W. Milonni, and J. R. Ackerhalt, J. Opt. Soc. Am. B 2, 130 (1985).

12. A. G. Gurtovnik, Izv. Vyssh. Uchebn. Zaved. Radiofiz. 1, 83 (1958).

13. A. V. Uspenskiy, Radio Eng. Electron. Phys. 8, 1145 (1963).

14. V. V. Korobkin and A. V. Uspenskiy, Sov. Phys. JETP 18, 693 (1964).

15. A. V. Uspenskiy, Radio Eng. Electron. Phys. 9, 605 (1964).

16. A. Z. Grasyuk and A. N. Orayevskiy, Radio Eng. Electron. Phys. 9, 424 (1964).

17. A. Z. Grasyuk and A. N. Orayevskiy, in Quantum Electronics and Coherent Light, P. A. Miles, ed. (Academic, New York, 1964), p. 192.

18. H. Haken, Z. Phys. 190, 327 (1966).

19. H. Risken, C. Schmid, and W. Weidlich, Z. Phys. 194, 337 (1966).

20. H. Risken and K. Nummedal, Phys. Lett. 26A, 275 (1968).

21. H. Risken and K. Nummedal, J. Appl. Phys. 39, 4662 (1968).

22. R. Graham and H. Haken, Z. Phys. 213, 420 (1968).

23. R. Graham, Phys. Lett. 58A, 440 (1976).

24. M. Renardy, Math. Methods Appl. Sci. 1, 194 (1979).

25. P. R. Gerber and M. Buttiker, Z. Physik B, 33, 219 (1979).

26. J. Zorell, Opt. Commun. 38, 127 (1981).

27. M. L. Minden and L. W. Casperson, IEEE J. Quantum Electron. QE-18, 1952 (1982).

28. S. T. Hendow and M. Sargent III, Opt. Commun. 43, 59 (1982).

29. P. Mandel and H. Zeghlache, Opt. Commun. 47, 146 (1983).

30. L. W. Casperson, Phys. Rev A 21, 911 (1980).

31. L. W. Casperson, Phys. Rev A 23, 248 (1981).

32. M. L. Minden and L. W. Casperson, IEEE J. Quantum Electron. QE-18, 1952 (1982).

33. M. L. Minden, "Modesplitting and coherent instabilities in high gain lasers," Ph.D. dissertation (University of California, Los Angeles, California, 1982).

34. S. T. Hendow and M. Sargent III, Opt. Commun. 40, 385 (1982).

35. P. Mandel, Opt. Commun. 44, 400 (1983).

36. P. Mandel, Opt. Commun. 45, 269 (1983).

37. L. A. Lugiato, L. M. Narducci, D. K. Bandy, and N. B. Abraham, Opt. Commun. 46, 115 (1983).

38. M. L. Minden and L. W. Casperson, J. Opt. Soc. Am. B 2, 120 (1985).

39. L. W. Hillman, R. W. Boyd, and C. R. Stroud, Opt. Lett. 7, 426 (1982).

40. E. N. Lorenz, J. Atmos. Sci. 20, 130 (1963).

41. J. L. Kaplan and J. A. Yorke, Commun. Math. Phys. 67, 93 (1979).

42. L. W. Casperson and A. Yariv, IEEE J. Quantum Electron. QE-8, 69 (1972). 